\title{
Further understanding of fat biology: Lessons from a fat fly
}

\author{
Joung-Woo Hong ${ }^{1}$ and Kye Won Park ${ }^{2,3}$ \\ ${ }^{1}$ Graduate School of East-West Medical Science \\ Kyung Hee University \\ Yongin 446-701, Korea \\ ${ }^{2}$ Department of Food Science and Biotechnology \\ Sungkyunkwan University \\ Suwon 440-746, Korea \\ ${ }^{3}$ Corresponding author: Tel, 82-31-290-7804; \\ Fax, 82-31-290-7882; E-mail, kwpark@ skku.edu \\ DOI 10.3858/emm.2010.42.1.007
}

Accepted 20 October 2009

Available Online 4 November 2009

Abbreviations: BAT, brown adipose tissue; C/EBP, CCAAT enhancer binding protein; DBD, DNA-binding domain; LBD, ligand-binding domain; PGC, PPAR $\gamma$ coactivator; PPAR $\gamma$, peroxisome proliferator activator receptor $\gamma$; PRDM16, PR domain containing 16; RXR, retinoid $\mathrm{X}$ receptor; WAT, white adipose tissue

\begin{abstract}
Obesity is a leading risk factor for insulin resistance, hypertension, hyperlipidemia, and cardiovascular complications, collectively referred to as metabolic diseases. Given the prevalence of obesity and its associated medical problems, new strategies are required to prevent or treat obesity and obesity-related metabolic effects. Here we summarize contributors of obesity, and molecular mechanisms controlling adipogenesis from studies in mammalian systems. We also discuss the possibilities of using Drosophila as a genetic model system to advance our understanding of players in fat biology.
\end{abstract}

Keywords: adipocytes; adipogenesis; adipose tissue; cell differentiation; Drosophila; models, biological; obesity; PPAR $\gamma$

\section{Obesity is the primary factor for metabolic disease}

Obesity, an excessive accumulation of body fat, is a chronic condition that contributes to a number of metabolic diseases, such as cardiovascular disease and type 2 diabetes (Kopelman, 2000). The prevalence of obesity is increasing worldwide, with as many as 400 million people being obese (Hofker and Wijmenga, 2009). As this rate is not showing any signs of declining in future, the need to develop anti-obesity medications is increasing. Although there have been numerous efforts in the development of anti-obesity drugs, the growth of obesity prevalence outruns the growth of the anti-obesity drug market, perhaps due to lack of molecular targets and strategies. Therefore, a better understanding of the molecular mechanisms controlling adipogenesis, especially in vivo, is required to identify new molecular targets and therapeutic interventions against obesity.

\section{What contributes to obesity?}

At least three determinants contribute to obesity. First, genetic factors, as seen in most human diseases, contribute to obesity. Mice studies indicate that genetic alterations can affect food intake, energy expenditure, and fat storage (Zhang et al., 1994; Tartaglia et al., 1995; Friedman and Halaas, 1998). Genetic predispositions associated with obesity or its related complications have also been identified in many human diseases, including Prader-Willi syndrome, Cohen's syndrome, lipodystrophy, and Carpenter's syndrome. These data demonstrate that multiple genes determine obese phenotypes in mice and humans.

Second, lifestyle including diet and exercise is another major contributor of obesity. Genetically identical, Pima Indians reside in two different regions: the Sierra Madre area of Mexico and in Arizona, USA. Among these individuals, Mexican Pima Indians are not overweight and have less diabetes, whereas Arizona Pima Indians are overweight and have a tendency for higher incidence of diabetes (Schulz et al., 2006). This study indicates that higher physical activity and less dietary fat intake by the Mexican residents compared to the Arizona Pima Indians prevents obesity and its related metabolic complications, despite the same genetic background.

Finally, genetic and environmental factors do not act independently; the interaction of genes and the environment determine obesity (West and York, 1998). For instance, two strains of mice can differ in their sensitivity to obesity induced by a high-fat diet, suggesting that genes and dietary environment act together to determine obesity phenotypes (Fisler et al., 1993; York et al., 1996). Therefore, 
genetic and environmental factors can affect bodyweight together through their interaction.

\section{Fat is stored in two distinct adipocytes}

Since obesity is essentially the amount of fat stored in fat cells called adipocytes, adipocytes are the primary focus in understanding this condition. Adipocytes have been classified into two types: white adipose tissue (WAT) and brown adipose tissue (BAT) (Spiegelman and Flier, 2001). WAT expresses the machinery of lipolysis, glucose uptake, and triglyceride synthesis. Thus, WAT is the primary site of energy storage. WAT also functions as an endocrine tissue, secreting adipokines such as leptin, adiponectin, and TNF $\alpha$ that regulates systemic metabolism (Tontonoz and Spiegelman, 2008). The importance of WAT in metabolism is shown by studies where WAT was the primary target tissue for the anti-diabetic drug, thiazolidinediones (Nolan et al., 1994). The efficacy of anti-diabetic drugs such as thiazolidinediones reveals that the proper regulation of triglyceride storage and the endocrine functions of WAT result in beneficial effects on glucose metabolism. By sharp contrast, BAT is specialized for energy expenditure through adaptive thermogenesis. BAT contains numerous mitochondria and uncoupling proteins (UCP) to generate heat (Spiegelman and Flier, 2001). Although BAT was not detected in adult humans for many years, recent studies show that brown adipocytes are dispersedly located and metabolically active (Nedergaard et al., 2007). Due to its distinct functions, BAT has been proposed by numerous groups as a prospective strategy to prevent obesity and its related complications (Park et al., 2008; Farmer, 2008). Better understanding of the molecular pathways that control WAT and BAT development may therefore provide new insight into adipocyte biology as well as therapeutic interventions for metabolic diseases.

\section{Molecular mechanisms controlling adipocyte differentiation are primarily revealed by studies using mammal systems}

To further understand fat biology, mechanisms controlling adipocyte differentiation should be considered. Preadipocyte cell lines have greatly facilitated the study of adipogenic programs. The white adipocyte cell lines, 3T3-L1 and 3T3-F442A, were derived from mouse embryonic fibroblasts. These cells develop into fat depots when injected into the peritoneum of nude mice, providing the identity of adipocyte precursors (Green and Meuth, 1974; Green and Kehinde, 1976). In 3T3-L1 cell culture systems, adipocyte differentiation starts with confluence induction, clonal expansion, growth arrest, to terminal differentiation. This process involves a cascade of activation of transcription factors. After confluence followed by growth arrest, hormonal induction (DMI: Dexamethasone, IBMX and insulin) initiates post confluence mitosis known as clonal expansion and acutely increases the expression of CCAAT-enhancer binding protein (C/EBP) $\beta$ and C/EBP $\delta$ transcription factors (Wu et al., 1995). These DMI induced C/EBP $\beta$ and C/EBP $\delta$ stimulate the critical regulators of adipocyte differentiation, peroxisome proliferator activator receptor (PPAR) $\gamma$ and C/EBP $\alpha$. PPAR $\gamma$ and $\mathrm{C} / \mathrm{EBP} \alpha$ terminally differentiate preadipocytes to adipocytes (Tontonoz and Spiegelman, 2008).

Brown and white adipocytes were initially considered to be generated from a common ancestor. $\beta$-Adrenergic stimulation or cold exposure forms brown adipocytes in WAT depots. Furthermore, PPAR $\gamma$ is required for both brown and white adipocytes. However, recent surprising studies have demonstrated that brown adipocytes derive from Myf-5 positive myogenic precursors distinct from precursor cells for white adipocytes (Seale et al., 2008). The transcription factor PR domain containing (PRDM)16 is shown to control the fate of myocytes and brown adipocytes in gain and loss of function studies. PRDM16 and PGC-1 $\alpha$ form a complex to drive brown adipocyte-specific programs by further stimulating PPAR $\gamma$ coactivator (PGC)-1 $\alpha$ functions in mitochondrial biogenesis and adaptive thermogenesis (Seale et al., 2008). The distinct differentiation programs and progenitors of brown and white fat cells could thus provide new strategies for the selective modulation of brown vs. white adipocytes to prevent obesity and metabolic diseases.

\section{The master regulator of adipogenesis, PPAR $\gamma$}

PPAR $\gamma$ is a member of the ligand-activated nuclear receptor superfamily, which functions as a transcription factor (Tontonoz and Spiegelman, 2008). It has been shown that PPAR $\gamma$ is a master regulator of adipogenesis and is the direct target of thiazolidinedione anti-diabetic drugs. The gained expression of PPAR $\gamma$ is sufficient to induce adipogenesis in fibroblasts, whereas PPAR $\gamma^{-1-}$ cells fail to differentiate into adipocytes in vitro and in vivo. Both mouse and human models have established that the loss of PPAR $\gamma$ is diabetogenic and causes lipodystrophy. PPAR $\gamma$ dimerizes with 
retinoid $X$ receptor (RXR) and drives the transcription of target genes when activated by ligands. Lipid metabolites such as eicosanoids from polyunsaturated fatty acids have been implicated and 15-deoxy- $\Delta 12,14$-prostaglandin $\mathrm{J} 2$ was also suggested as an endogenous ligand (Forman et al., 1995). However, the physiological relevance of these ligands in adipocytes remains unclear. Thiazolidinediones are synthetic PPAR $\gamma$ ligands and are widely used to study the function of PPAR $\gamma$ related gene expression or adipocyte differentiation (Lehmann et al., 1995). Activated PPAR $\gamma$ induces numerous target genes such as aP2 (fatty acid binding protein), LPL (lipoprotein lipase), CD36 (fatty acid transporter), Glut4 (glucose transporter), adiponectin, and adipogenic programs (Lee et al., 2008).

In the absence of ligand, PPAR $\gamma$ and repressor complexes can bind to target genes. Those repressors including nuclear receptor corepressor (NCoR), silencing mediator for retinoid and thyroid receptors (SMART), and Sirtuins act as negative regulators of adipogenesis (Lefterova and Lazar, 2009). In mammals, sirtuin homolog, SIRT1 regulates fat mobilization by inhibiting PPAR $\gamma$ (Picard et al., 2004). SIRT1 also binds and deacetylates Forkhead box class $O$ (FOXO) and PGC-1 $\alpha$ to modulate glucose and insulin homeostasis (Finkel et al., 2009). AMP-activated protein kinase (AMPK) regulates glucose and fatty acid metabolism and acts as a mediator of leptin and adiponectin signaling. Since both AMPK and SIRT can play a role in energy sensors, they are considered as potential targets of energy expenditure against PPAR $\gamma$ activation and obesity. Therefore, it is conceivable that the integration of multiple signaling systems may also contribute to PPAR $\gamma$ activity.

Current therapeutic efforts are focusing on the modulation of PPAR $\gamma$ activation. However, one fundamental question of PPAR $\gamma$ biology remains to be solved. PPAR $\gamma$ expression is highly regulated during adipogenesis and most abundantly found in the adipose tissues. What are upstream transcriptional regulators that induce PPAR $\gamma$ expression? C/EBPs are major stimulators of PPAR $\gamma$ expression, as indicated by studies in mice lacking $\mathrm{C} / \mathrm{EBP} \beta$ and $\mathrm{C} / \mathrm{EBP} \delta$ that have shown defects in adipocyte development. However, PPAR $\gamma$ expression is still detected in these mice suggesting that other in vivo transcriptional systems play roles in the modulation of PPAR $\gamma$ expression (Tanaka et al., 1997). Other transcription factors such as Krox-20, KLFs, EBF, Id2, and ADD1/SREBP1c have been reported to promote differentiation and PPAR $\gamma$ expression. Conversely, KLF2, GATA2/3, HES-1,
IRF, and TCF/LEF are shown to inhibit differentiation (Lefterova and Lazar, 2009).

Besides the lack of full understanding for the upstream regulators of PPAR $\gamma$, transcription factors that contribute to the fat-specific expression of PPAR $\gamma$ also remain elusive. A PPAR $\gamma$ gene produces two transcripts of PPAR $\gamma 1$ and PPAR $\gamma 2$ from alternative promoters. Both isoforms are abundantly expressed in adipocytes, with more adipocyte-specific expression of the $\gamma 2$ isoform. This expression pattern raises an outstanding question regarding adipose-specific PPAR $\gamma$ regulation for future studies. Previous data have shown that adipose-specific stimulation of PPAR $\gamma$ expression by a small molecule ameliorated metabolic conditions in diabetic mice by the lowering glucose level, the reduction of macrophage infiltration in fat tissues, lowering triglyceride, and free fatty acids in circulation (Waki et al., 2007). Therefore, tissue specific regulators of PPAR $\gamma$ expression will potentially provide windows of opportunities for therapeutic intervention. The identification of these factors will help to further understand PPAR $\gamma$ and adipocyte biology. To this end, more efficient systems are required to successfully screen in vivo modulators of adipocyte development and determine contributors of obesity. In the next section, we will discuss the possibilities of using an alternative genetic model system to screen in vivo modulators.

\section{The fly as alternative system for fat biology?}

Despite significant advances in understanding adipogenesis using in vitro cell culture systems and mice models, several gaps still remain to be filled. Firstly, what key factors regulate PPAR $\gamma$ expression in particular time and space? And secondly, what PPAR $\gamma$ target genes are required for adipocyte differentiation? Invertebrate systems such as $C$. elegans and $D$. melanogaster have been powerful models to identify and analyze genes essential to human diseases due to their genetic versatility, the functional analogy to higher organisms, and ease to utilize (Culetto and Sattelle, 2000; Bier, 2005). Unfortunately, these advantages have not yet been fully applied to studies on obesity and lipid metabolism at least partly due to concerns that differences exist in fat biology between invertebrate and mammalian systems (Rosen et al., 2000). For instance, the fat in worms and flies is not deposited in dedicated adipocytes but in multifunctional organs such as the fat body. However, a couple of lines of studies strongly support the notion that invertebrates can be efficient model systems to provide useful infor- 
mation on fat storage and utilization by mimicking pivotal metabolic processes observed in mammals (discussed below).

As a possible genetic system to further dissect fat biology, we will primarily focus on fly systems. In flies, the masses and sheets of adipose tissue that are distributed throughout the organism are collectively called the fat body (Bharucha, 2009). Like mammalian adipocytes, fat body cells function as a major fat depot, storing triglyceride in intracellular lipid droplets. Upon starvation, accumulated triglyceride in fat body cells is converted to fatty acids, which in turn are metabolized to produce ketone bodies. These metabolites are then released into the circulation for use as fuel until the next feeding. The conversion of triglyceride to fatty acid is catalyzed by a triacylglycerol lipase, brummer (bmm), a homolog of human triacylglycerol lipase (ATGL) (Gronke et al., 2005). bmm was identified by genome-wide transcriptome analysis in adult flies that were fed and fasted. Phylogenetic analysis indicates that this BMM lipase is evolutionarily well conserved from fly to human. bmm loss- and gain-of-function mutants show embryonic lethality and an increased amount of organismal triglyceride relative to protein content, as well as an increase size and number of lipid storage droplets in single fat body cells, respectively, which are reminiscent of the role of ATGL in humans (Zimmermann et al., 2004). In addition, enzymatic cascades for triglyceride synthesis are also conserved in all organisms. This conserved function implies that fundamental components and regulated mechanisms of lipid storage and utilization are also evolutionarily conserved. More recently, it was shown that another conserved gene, Adipose ( $A d p)$, exerts an anti-obesity function as a core of the corepressor complex in worms, flies, and mice (Suh et al., 2007). Adp was initially isolated from naturally occurring obese flies where both alleles were mutagenized (Doane, 1960, 1969). Molecular and genetic analyses represent that ADP seems to inhibit lipid accumulation in a dose-sensitive manner by recruiting histone deacetylase (HDAC)3 to PPAR $\gamma$, and thereby inhibiting PPAR $\gamma$ activity. Like other members of nuclear hormone receptors, in the absence of a ligand, PPAR $\gamma$ represses transcription by recruiting corepressor complexes containing HDACs and other chromatin modifying enzymes that make nearby chromatin structures condense (Guan et al., 2005). Interestingly, the overexpression of $A d p$ in the fat bodies of transgenic flies causes reduced fat storage (the amount of triglyceride) and decreased fat body size, which are analogous to characteristic features identified in $A d p$ transgenic mice and cultured cells. These findings suggest that not only is a controlled balance between lipogenesis and lipolysis (triglyceride mobilization) evolutionarily conserved, but that PPAR $\gamma$ activity (target of ADP) is critical for fat storage as well.

\section{Does a fly really have PPAR $\gamma$ ?}

Intriguingly, it has long been widely accepted that flies do not have an obvious homolog of PPAR $\gamma$. As always, one answered question raises another question: how does Adp work in flies without PPAR $\gamma$ ? Although it is not easy to interpret the phenotypes observed in Adp transgenic flies with the inclusion, the absence of a fly PPAR $\gamma$ homolog also makes alternative explanations simultaneously plausible. A possibility has not been experimentally ruled out that a divergent but bi- or multi-functional protein, frequently observed in lower organisms, may act as a functional substituent of PPAR $\gamma$ in flies. The claim that flies do not have a homolog of PPAR $\gamma$ is solely based on a simple BLAST search. Indeed, a search performed with a human PPAR $\gamma$ sequence in the uniGene database demonstrates that worm Nuclear Hormone Receptor (NHR)-23 has $50 \%$ identity with human PPAR $\gamma$; however, no fly protein obtains a statistically significant score

Table 1. Comparison of PPAR $\gamma$ s in the uniGene database.

\begin{tabular}{lllcr}
\hline GeneBank Accession \# & \multicolumn{1}{c}{ Reference Protein Name } & Species & Identity (\%) & Length (AA) \\
\hline NP_056953.2 & PPAR $\gamma$, isoform 2 & H. sapiens & 100 & 504 \\
NP_035276.2 & PPAR $\gamma$, isoform 2 & M. musculus & 98.4 & 504 \\
NP_571542.1 & PPAR, partial & D. rerio & 76.8 & 331 \\
NP_001088831.1 & hypothetical protein LOC496107 & X. laevis & 68.7 & 395 \\
NP_001020976.1 & Nuclear Hormone Receptor family member (nhr-23) & C. elegans & 50 & 96 \\
\hline
\end{tabular}

The uniGene is a database of transcriptomes where individual entries consists of a group of transcripts that seem to originate from an identical or similar transcription locus, i.e. gene or expressed pseudogene, meaning that information on protein similarities, gene expression, cDNA clones, and genomic location is included. Thus, a higher value of uniGene Identity suggests not only higher similarity to the query sequence but also more comprehensive likelihood to be a functional homolog of the query species. The search results shown below were performed with protein sequences of the hPPAR $\gamma$ isoform 2. 
Table 2. Protein similarities of PPAR $\gamma$ s in the GeneBank Protein database.

\begin{tabular}{|c|c|c|c|c|}
\hline GeneBank Accession \# & Reference Protein Name & Species & Identity (\%) & Length (aa) \\
\hline NP_056953.2 & PPAR $\gamma$, isoform 2 & H. sapiens & 100 & 505 \\
\hline NP_035276.2 & PPAR $\gamma$, isoform 2 & M. musculus & 96 & 505 \\
\hline NP_571542.1 & PPAR $\gamma$ & D. rerio & 67 & 527 \\
\hline NP_001088831.1 & hypothetical protein LOC496107 & $X$. laevis & 54 & 469 \\
\hline AAN11687.1 & Ecdysone-induced protein $75 \mathrm{~B}$, isoform $\mathrm{C}$ & D. melanogaster & 27 & 1199 \\
\hline AAN11687.1 & Ecdysone-induced protein $75 \mathrm{~B}$, isoform C, $1-560$ aa & D. melanogaster & 31 & 560 \\
\hline NP_001020976.1 & Nuclear Hormone Receptor family member (nhr-23) & C. elegans & 24 & 553 \\
\hline
\end{tabular}

Comparisons with the human PPAR $\gamma$ protein sequence in the GeneBank Protein database reveal putative sequences of statistically significant similarities in all Metazoan species. Higher percentiles of identity suggests more likelihood of each protein to be a homolog of human PPAR $\gamma$ protein.

(Table 1). UniGene is a database of transcriptomes and contains various information including genomic location, expression pattern, and cDNA on a group of transcripts that seem to be originated from an identical or similar transcription locus (Wheeler et al., 2003). Accordingly, a higher value in uniGene identity suggests not only higher sequence similarities with the query but also more comprehensive likelihood to be a functional homolog of the query species. The result therefore suggests that a fly does not have a protein whose characteristic features are closely related to PPAR $\gamma$.

However, it is unlikely that this failure to obtain a score inevitably means that a fly does not have even a "fly version" of PPAR $\gamma$ by several points. First, when a similar search was performed in the BLAST protein database, an ecdysone-induced protein E75 (E75) was identified to bear 27\% identity with human PPAR $\gamma$, which is even higher than the worm NHR-23 (24\%) (Table 2). This discordance between the results of the two searches is probably due to dynamic expression patterns and an unusually large size of E75. As mentioned, computation of a uniGene identity relies not only on sequences but also on a variety of other information including spatiotemporal expression patterns. E75, a nuclear receptor member of the ligand-regulated transcription factor superfamily, is involved in numerous biological events such as ecdysis, ecdysteroid metabolism, cuticle formation, antimicrobial humoral responses, and oogenesis, indicating that its expression pattern is dynamic (Kozlova and Thummel, 2000; Maglich et al., 2001; Bialecki et al., 2002; Kleino et al., 2005). Additionally, the size of the E75 gene product is much larger than those of other homologs: E75 is composed of 1199 amino acids whereas others are 501 amino acids on average. The additional amino acids occupy the C-terminal half of E75, and upon removal of the last 640 amino acids, the sequence identity of the truncated E75 increases 15\%. Collectively, its dynamic expression pattern and unusually large size cause E75 to have some penalties in score calculation. Second, members of the nuclear-receptor superfamily are defined by the presence of a highly conserved DNA-binding domain (DBD) and a less conserved C-terminal ligand-binding domain (LBD) (King-Jones and Thummel, 2005). Like others, E75 has well-defined DBD and LBD within the first half of the protein (Segraves and Hogness, 1990). Pair-wise alignment shows that human PPAR $\gamma$ and E75 share significant conservation between their DBDs $(69 \%$ in identity) but LBDs seem to be relatively divergent (28\% in identity) (Figure 1$)$. The DBD of PPAR $\gamma$ comprises a $\mathrm{C} 4$ zinc finger whose canonical consensus sequence, $\mathrm{CX}_{2} \mathrm{CX}_{13} \mathrm{CX}_{2} \mathrm{CX}_{16}$ $\mathrm{CX}_{3} \mathrm{CX}_{9} \mathrm{CX}_{2} \mathrm{C}$, is completely identical with that of E75 (Figure 1B, top). In contrast to DNA binding affinity, it has been shown that this $\mathrm{C} 4$ zinc finger is a pivotal determinant of the target specificity of the nuclear receptor superfamily (Umesono and Evans, 1989), raising the possibility that E75 may recognize and bind to a similar or identical sequence element to which PPAR $\gamma$ binds. Consistent with the definition of the nuclear-receptor superfamily, the LBD of E75 appears to be quite different from that of human PPAR $\gamma$. Accordingly, it remains elusive whether the lower similarity between LBDs indicates the existence of a unique ligand(s) of E75 distinct from a PPAR $\gamma$ 's ligand(s). However, the structural conservation of the two signature domains of E75 provides support for a putative role of E75 as a functional understudy of PPAR $\gamma$. Finally, the high-affinity binding of PPAR $\gamma$ to DNA requires heterodimerization with $R X R$, a universal heterodimeric partner of the nuclear hormone receptor superfamily (Allenby et al., 1993). Like mammals, ultraspiracle (USP), a fly homolog of $\mathrm{RXR}$, has been demonstrated to form heterodimers with various family members including the ecdysone receptor (EcR) (Oro et al., 1990; Yao et al., 1992). Interestingly, RXR is able to successfully heterodimerize with EcR for DNA binding and 
A

$\begin{array}{lcclcc} & \text { HPPAR } \gamma_{-} D B D & \text { E75c_DBD } & & \text { HPPAR } \gamma_{-} L B D & \text { E75c_LBD } \\ \text { hPPAR_DBD } & 1.0000 & 0.6892 & \text { HPPAR } \gamma_{-} L B D & 1.0000 & 0.2849 \\ \text { E75c_DBD } & 0.6892 & 1.0000 & \text { E75c_LBD } & 0.2849 & 1.0000\end{array}$

B

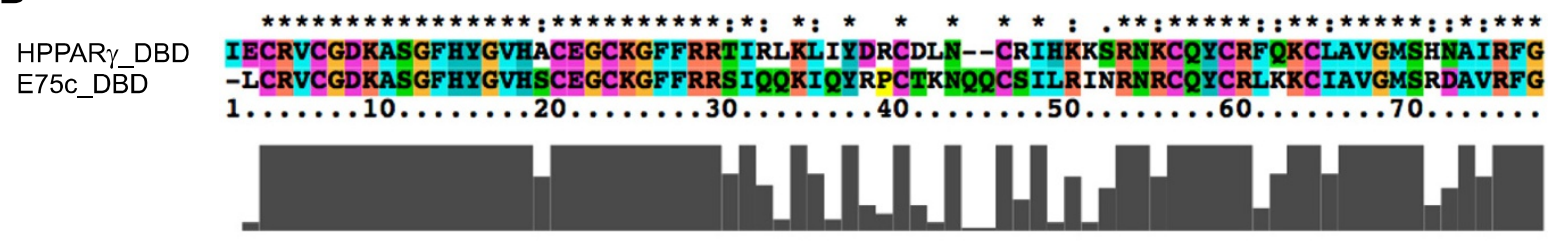
(1)

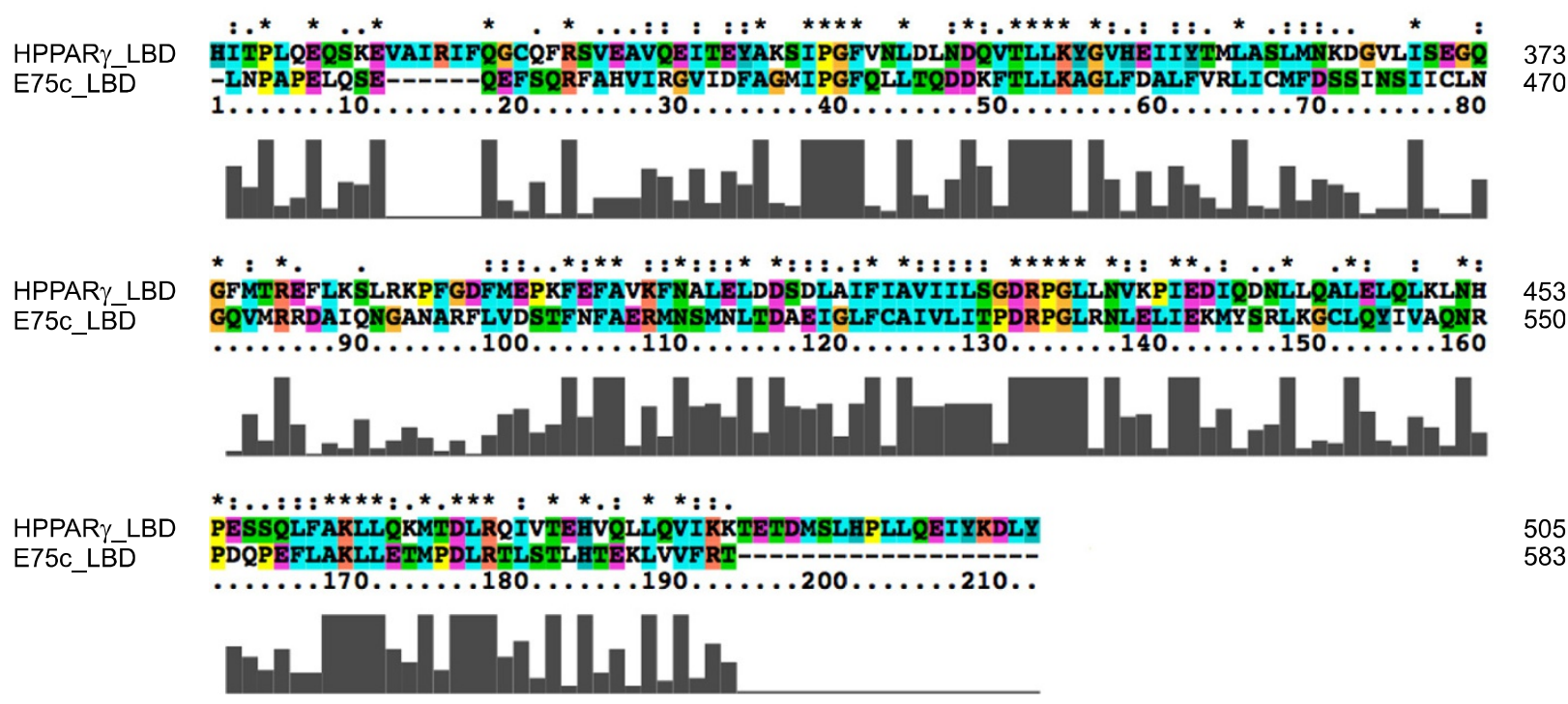

Figure 1. Comparison of protein sequences between human PPAR $\gamma$ and the fly ecdysone-induced protein $E 75$ isoform $C$ (E75C). The two sequences were aligned by ClustalX v2.0.10 with the protein weight matrix, Gonnet series, and then the alignments and the sequence identities were analyzed and calculated by Pfaat v2.0 (Pfizer Global Research and Development) (Caffrey et al., 2007). DBD: PPARy 137-211 aa, E75c 243-319 aa. LBD: PPARy 237-504 aa, E75c 396-583 aa. (A) Sequence identity between characteristic domains of human PPAR $\gamma$ and fly E75c. The DNA-binding domains (DBDs) between them display $69 \%$ identity but the ligand-binding domains (LBDs) are relatively less conserved ( $28 \%$ in identity). (B) Sequence alignments of DBDs (Top) and LBDs (Bottom three) between PPAR $\gamma$ and E75c. "* " indicates positions that have a single, fully conserved residue. ": "indicates that one of the following 'strong' groups is fully conserved: STA, NEQK, NHQK, NDEQ, QHRK, MILV, MILF, HY, FYW (single amino acid code). ". " indicates that one of the following 'weaker' groups is fully conserved: CSA, ATV, SAG, STNK, STPA, SGND, SNDEQK, NDEQHK, NEQHRK, FVLIM, HFY (single amino acid code). These are all the positively scoring groups that occured in the Gonnet Pam250 matrix. The strong and weak groups are defined as a strong score $>0.5$ and a weak score $\leq 0.5$, respectively. Gray bars under the alignments represent the conservation score in an arbitrary unit.

transcriptional activation (Thomas et al., 1993). Recently, a 'ligand-sensor system identified a new ligand of USP and showed the involvement of USP in the response to nutrients and metabolites (Palanker et al., 2006). These findings suggest that at least the transcriptional regulation of the nuclear receptor via heterodimerization with USP is evolutionarily conserved even if E75 is not an actual homolog of PPAR $\gamma$. Taken together, it is conceivable that fly E75 could act as a divergent functional homolog of PPAR $\gamma$, although their similarity has been masked by functional diversity and structural heterogeneity.
One may argue that E75 is not a genuine dedicator to adipogenesis and lipid metabolism in flies at least partly because of its multi-functionality and poor overall sequence homology. In fact, it is believed that E75 is more closely related to vertebrate REV-ERB (Marvin et al., 2009), and a recent study suggests an unexpected function of E75 as a diatomic gas sensor via a heme prosthetic group in its ligand binding pocket (Reinking et al., 2005). However, the versatility of E75 fuels speculation that it acts as an actual fly version of PPAR $\gamma$ in addition to the pre-identified roles. This speculation is based on the "duplica- 
tion-degeneration-complementation (DDC)" model where each of a paralogous gene pair produced after gene duplication accumulates a loss-of-function, resulting in subfunctionalization of the common ancestral gene and thereby a functional complement by the other copy (Force et al., 1999). Examples of the DDC model are frequently observed in low organisms. For instance, the nervous systems of humans and flies display tremendous conservation in function and structure, but nevertheless two families of major trophic factors, neurotrophin and neuregulin, and their corresponding receptors are not identified in flies (Hidalgo et al., 2006). Instead, some epidermal growth factors (EGFs) and their receptors act as functional homologs of neuregulin in certain contexts (Hidalgo et al., 2006). More recently, spätzle (spz) 2, also called Drosophila Neurotrophin 1 (DNT1), was identified as the first homolog of neurotrophin in flies (Zhu et al., 2008). It has been known that $s p z 2$ is a divergent member of the spätzle ligand family that plays a role as a ligand of Toll receptors involved in immune responses in flies (Parker et al., 2001). Therefore, the multiple functions of a certain protein in low organisms cannot always be utilized as a barometer to determine whether it is a functional homolog of a protein of interest but rather may be an unrevealed clue for identifying the masked function.

\section{Can we take advantage of the fly system to study fat biology?}

Fly genetic systems may also be useful to further dissect PPAR $\gamma$ biology. Recent studies have shown that insulin/IGF functions are conserved in $D$. melanogaster. Ablation of insulin producing cells yields diabetic phenotypes. Geminard et al. (2009), further demonstrated that a signal from fat cells in flies controls insulin-like peptides secretion from insulin producing cells. Furthermore, Sulfonylurea, a drug used against diabetic conditions in human, also affects sugar concentration in flies. Together, these data suggest that similar mechanisms regulate glucose homeostasis in flies. PPAR $\gamma$ interacts with energy storaging/ expenditure genes including SIRT1 and PGC-1 $\alpha$ in mammals. Therefore, genetic interaction between $P P A R \gamma$ and other energy storaging/expenditure genes such as FOXO and AMPK besides SIRT1 and PGC-1 $\alpha$ can be potentially studied using this system. Similarly, epistasis analysis between two major players in metabolism, SIRT1 and AMPK can be used to determine the functional order of action of these two genes. Thus, fly genetics toward understanding of obesity is valuable tools to identify the function and mechanisms of the players in vivo.

\section{Future perspective}

Obesity is a devastating threat to our modern society, causing urgent demands for therapeutics to combat it. The development of strategies to oppose the obesity epidemic requires us to better understand the contributing factors of obesity. However, this requirement has frequently been hampered by the absence of an effective tool(s) for identifying in vivo candidates, primarily due to the innate limitations of mammalian systems with which most research on obesity and fat biology have been performed. Thus, forthcoming studies on fly PPARy will not only throw light on understanding the fundamentals of fat biology across species, but will also suggest ways to develop anti-obesity medications.

Although several tiers of evidence are supporting the speculation that a functional homolog of PPAR $\gamma$ exists in flies, it does not exclude the possibility that the fly has evolved its own unique systems for adipogenesis and lipid metabolism quite distinct from mammals. It is also possible to anticipate that both are not mutually exclusive. Therefore, determining whether flies have a functional counterpart of mammal PPAR would be a good starting point to better understand the details of fat biology in flies. However, whether a fly has a PPAR $\gamma$ homolog or its own unique adipogenic system, genetic screening for adipogenesis will be required to further understand the in vivo players of fat biology. In any case, the attempt is worth performing to devise a novel means for more efficiently solving many of the unsolved questions in the field.

\section{Acknowledgements}

This work was supported by a grant from the Kyung Hee University in 2009. (KHU-20090751).

\section{References}

Allenby G, Bocquel MT, Saunders M, Kazmer S, Speck J, Rosenberger M, Lovey A, Kastner P, Grippo JF, Chambon P, Levin AA. Retinoic acid receptors and retinoid $X$ receptors: interactions with endogenous retinoic acids. Proc Natl Acad Sci USA 1993;90:30-4

Bharucha KN. The epicurean fly: using Drosophila melanogaster to study metabolism. Pediatr Res 2009;65: 
132-7

Bialecki M, Shilton A, Fichtenberg C, Segraves WA, Thummel CS. Loss of the ecdysteroid-inducible E75A orphan nuclear receptor uncouples molting from metamorphosis in Drosophila. Dev Cell 2002;3:209-20

Bier E. Drosophila, the golden bug, emerges as a tool for human genetics. Nat Rev Genet 2005;6:9-23

Caffrey DR, Dana PH, Mathur V, Ocano M, Hong EJ, Wang YE, Somaroo S, Caffrey BE, Potluri S, Huang ES. PFAAT version 2.0: a tool for editing, annotating, and analyzing multiple sequence alignments. BMC Bioinformatics 2007;8: 381

Culetto E, Sattelle DB. A role for Caenorhabditis elegans in understanding the function and interactions of human disease genes. Hum Mol Genet 2000;9:869-77

Doane WW. Developmental physiology of the mutant female sterile(2)adipose of Drosophila melanogaster. I. Adult morphology, longevity, egg production, and egg lethality. $J$ Exp Zool 1960;145:1-21

Doane WW. Amylase variants in Drosophila melanogaster: linkage studies and characterization of enzyme extracts. J Exp Zool 1969;171:31-41

Farmer SR. Molecular determinants of brown adipocyte formation and function. Genes Dev 2008;22:1269-75

Finkel T, Deng CX, Mostoslavsky R. Recent progress in the biology and physiology of sirtuins. Nature 2009;460:587-91

Fisler JS, Warden CH, Pace MJ, Lusis AJ. BSB: a new mouse model of multigenic obesity. Obes Res 1993;1:271-80

Force A, Lynch M, Pickett FB, Amores A, Yan YL, Postlethwait J. Preservation of duplicate genes by complementary, degenerative mutations. Genetics 1999; 151:1531-45

Forman BM, Tontonoz P, Chen J, Brun RP, Spiegelman BM, Evans RM. 15-Deoxy-delta 12, 14-prostaglandin $\mathrm{J} 2$ is a ligand for the adipocyte determination factor PPAR gamma. Cell 1995;83:803-12

Friedman JM, Halaas JL. Leptin and the regulation of body weight in mammals. Nature 1998;395:763-70

Geminard C, Rulifson EJ, Leopold P. Remote control of insulin secretion by fat cells in Drosophila. Cell Metab 2009;10:199-207

Green H, Meuth M. An established pre-adipose cell line and its differentiation in culture. Cell 1974;3:127-33

Green $\mathrm{H}$, Kehinde $\mathrm{O}$. Spontaneous heritable changes leading to increased adipose conversion in 3T3 cells. Cell 1976;7:105-13

Gronke S, Mildner A, Fellert S, Tennagels N, Petry S, Muller $\mathrm{G}$, Jackle H, Kuhnlein RP. Brummer lipase is an evolutionary conserved fat storage regulator in Drosophila. Cell Metab 2005;1:323-30

Guan HP, Ishizuka T, Chui PC, Lehrke M, Lazar MA. Corepressors selectively control the transcriptional activity of PPARgamma in adipocytes. Genes Dev 2005;19:453-61

Hidalgo A, Learte AR, Mcquilton P, Pennack J, Zhu B.
Neurotrophic and gliatrophic contexts in Drosophila. Brain Behav Evol 2006;68:173-80

Hofker M, Wijmenga C. A supersized list of obesity genes. Nat Genet 2009;41:139-40

King-Jones K, Thummel CS. Nuclear receptors--a perspective from Drosophila. Nat Rev Genet 2005;6:311-23

Kleino A, Valanne S, Ulvila J, Kallio J, Myllymaki H, Enwald $H$, Stoven S, Poidevin M, Ueda R, Hultmark D, Lemaitre B, Ramet M. Inhibitor of apoptosis 2 and TAK1-binding protein are components of the Drosophila Imd pathway. EMBO J 2005;24:3423-34

Kopelman PG. Obesity as a medical problem. Nature 2000;404:635-43

Kozlova T, Thummel CS. Steroid regulation of postembryonic development and reproduction in Drosophila. Trends Endocrinol Metab 2000;11:276-80

Lee KJ, Ha ES, Kim MK, Lee SH, Suh JS, Lee SH, Park KH, Park JH, Kim DJ, Kang D, Kim BC, Jeoung D, Kim YK, Kim $\mathrm{HD}$, Hahn JH. CD36 signaling inhibits the translation of heat shock protein 70 induced by oxidized low density lipoprotein through activation of peroxisome proliferators-activated receptor gamma. Exp Mol Med. 2008;40:658-68

Lefterova MI, Lazar MA. New developments in adipogenesis. Trends Endocrinol Metab 2009;20:107-14

Lehmann JM, Moore LB, Smith-Oliver TA, Wilkison WO, Willson TM, Kliewer SA. An antidiabetic thiazolidinedione is a high affinity ligand for peroxisome proliferator-activated receptor gamma (PPAR gamma). J Biol Chem 1995;270: 12953-6

Maglich JM, Sluder A, Guan X, Shi Y, Mckee DD, Carrick K, Kamdar K, Willson TM, Moore JT. Comparison of complete nuclear receptor sets from the human, Caenorhabditis elegans and Drosophila genomes. Genome Biol 2001;2: RESEARCH0029

Marvin KA, Reinking JL, Lee AJ, Pardee K, Krause HM, Burstyn JN. Nuclear receptors homo sapiens Rev-erbbeta and Drosophila melanogaster E75 are thiolate-ligated heme proteins which undergo redox-mediated ligand switching and bind CO and NO. Biochemistry 2009;48:7056-71

Nedergaard J, Bengtsson T, Cannon B. Unexpected evidence for active brown adipose tissue in adult humans. Am J Physiol Endocrinol Metab 2007;293:E444-52

Nolan JJ, Ludvik B, Beerdsen P, Joyce M, Olefsky J. Improvement in glucose tolerance and insulin resistance in obese subjects treated with troglitazone. N Engl J Med 1994;331:1188-93

Oro AE, Mckeown M, Evans RM. Relationship between the product of the Drosophila ultraspiracle locus and the vertebrate retinoid X receptor. Nature 1990;347:298-301

Palanker L, Necakov AS, Sampson HM, Ni R, Hu C, Thummel $\mathrm{CS}$, Krause HM. Dynamic regulation of Drosophila nuclear receptor activity in vivo. Development 2006;133:3549-62

Park KW, Halperin DS, Tontonoz P. Before they were fat: adipocyte progenitors. Cell Metab 2008;8:54-7

Parker JS, Mizuguchi K, Gay NJ. A family of proteins related 
to Spatzle, the toll receptor ligand, are encoded in the Drosophila genome. Proteins 2001;45:71-80

Picard F, Kurtev M, Chung N, Topark-Ngarm A, Senawong T, Machado DE, Oliveira R, Leid M, Mcburney MW, Guarente L. Sirt1 promotes fat mobilization in white adipocytes by repressing PPAR-gamma. Nature 2004;429:771-6

Reinking J, Lam MM, Pardee K, Sampson HM, Liu S, Yang P, Williams S, White W, Lajoie G, Edwards A, Krause HM. The Drosophila nuclear receptor e75 contains heme and is gas responsive. Cell 2005;122:195-207

Rosen ED, Walkey CJ, Puigserver P, Spiegelman BM. Transcriptional regulation of adipogenesis. Genes Dev 2000;14:1293-307

Schulz LO, Bennett PH, Ravussin E, Kidd JR, Kidd KK, Esparza J, Valencia ME. Effects of traditional and western environments on prevalence of type 2 diabetes in Pima Indians in Mexico and the U.S. Diabetes Care 2006;29:1866-71

Seale P, Bjork B, Yang W, Kajimura S, Chin S, Kuang S, Scime A, Devarakonda S, Conroe HM, Erdjument-Bromage $\mathrm{H}$, Tempst P, Rudnicki MA, Beier DR, Spiegelman BM. PRDM16 controls a brown fat/skeletal muscle switch. Nature 2008;454:961-7

Segraves WA, Hogness DS. The E75 ecdysone-inducible gene responsible for the $75 \mathrm{~B}$ early puff in Drosophila encodes two new members of the steroid receptor superfamily. Genes Dev 1990;4:204-19

Spiegelman BM, Flier JS. Obesity and the regulation of energy balance. Cell 2001;104:531-43

Suh JM, Zeve D, Mckay R, Seo J, Salo Z, Li R, Wang M, Graff $\mathrm{JM}$. Adipose is a conserved dosage-sensitive antiobesity gene. Cell Metab 2007;6:195-207

Tanaka T, Yoshida N, Kishimoto T, Akira S. Defective adipocyte differentiation in mice lacking the C/EBPbeta and/or C/EBPdelta gene. EMBO J 1997;16:7432-43

Tartaglia LA, Dembski M, Weng X, Deng N, Culpepper J, Devos R, Richards GJ, Campfield LA, Clark FT, Deeds J, Muir C, Sanker S, Moriarty A, Moore KJ, Smutko JS, Mays GG, Wool EA, Monroe CA, Tepper RI. Identification and expression cloning of a leptin receptor, OB-R. Cell 1995;83:1263-71

Thomas HE, Stunnenberg HG, Stewart AF. Heterodi- merization of the Drosophila ecdysone receptor with retinoid $X$ receptor and ultraspiracle. Nature 1993;362:471-5

Tontonoz P, Spiegelman BM. Fat and beyond: the diverse biology of PPARgamma. Annu Rev Biochem 2008;77: 289-312

Umesono K, Evans RM. Determinants of target gene specificity for steroid/thyroid hormone receptors. Cell 1989;57:1139-46

Waki H, Park KW, Mitro N, Pei L, Damoiseaux R, Wilpitz DC, Reue K, Saez E, Tontonoz P. The small molecule harmine is an antidiabetic cell-type-specific regulator of PPARgamma expression. Cell Metab 2007;5:357-70

West DB, York B. Dietary fat, genetic predisposition, and obesity: lessons from animal models. Am J Clin Nutr 1998;67:505S-512S

Wheeler DL, Church DM, Federhen S, Lash AE, Madden TL, Pontius JU, Schuler GD, Schriml LM, Sequeira E, Tatusova TA, Wagner L. Database resources of the National Center for Biotechnology. Nucleic Acids Res 2003;31:28-33

Wu Z, Xie Y, Bucher NL, Farmer SR. Conditional ectopic expression of C/EBP beta in NIH-3T3 cells induces PPAR gamma and stimulates adipogenesis. Genes Dev 1995;9: 2350-63

Yao TP, Segraves WA, Oro AE, Mckeown M, Evans RM. Drosophila ultraspiracle modulates ecdysone receptor function via heterodimer formation. Cell 1992;71:63-72

York B, Lei K, West DB. Sensitivity to dietary obesity linked to a locus on chromosome 15 in a CAST/Ei x C57BL/6J F2 intercross. Mamm Genome 1996;7:677-81

Zhang Y, Proenca R, Maffei M, Barone M, Leopold L, Friedman JM. Positional cloning of the mouse obese gene and its human homologue. Nature 1994;372:425-32

Zhu B, Pennack JA, Mcquilton P, Forero MG, Mizuguchi K, Sutcliffe B, Gu CJ, Fenton JC, Hidalgo A. Drosophila neurotrophins reveal a common mechanism for nervous system formation. PLoS Biol 2008;6:e284

Zimmermann R, Strauss JG, Haemmerle G, Schoiswohl G, Birner-Gruenberger R, Riederer M, Lass A, Neuberger G, Eisenhaber F, Hermetter A, Zechner R. Fat mobilization in adipose tissue is promoted by adipose triglyceride lipase. Science 2004;306:1383-6 